\title{
Beating the limitation of energy gap law utilizing deep red MR-TADF
}

\section{emitter with narrow energy-bandwidth}

Yuewei Zhang ${ }^{1,2}$, Dongdong Zhang ${ }^{1,2}$, Tianyu Huang ${ }^{1,2}$, Guomeng Li $^{1,2}$, Chen Zhang ${ }^{1,2}$, Yang

$$
\text { Lu, Ziyang Liu }{ }^{1,2} \text { and Lian Duan }{ }^{1,2 *}
$$

The development of high-performance deep red/near-infrared organic light-emitting diodes is hindered by strong non-radiative processes as governed by the energy gap law. Herein, a novel BN-containing skeleton featuring linear $\mathrm{N}-\pi-\mathrm{N}$ and $\mathrm{B}-\pi-\mathrm{B}$ structure is developed, establishing partial bonding/antibonding character on phenyl core for enhanced electronics coupling of para-positioned $\mathrm{B}$ atoms as well as $\mathbf{N}$ atoms to narrow energy gaps. Also, the remained MR effect on the peripheral skeleton to maintain the MR effect to minimize the bonding/ antibonding character and suppress vibrational coupling between $\mathrm{S}_{0}$ and $S_{1}$, thereby fundamentally overcoming the luminescent boundary set by the energy gap law. The target molecules R-BN and R-TBN exhibited extremely high PLQYs of $100 \%$ with emission wavelengths at 666 and $686 \mathrm{~nm}$, respectively. The narrow FWMHs of $38 \mathrm{~nm}$ observed also testify the effectiveness of vibronic suppression. The corresponding OLEDs afford record-high EQEs over $28 \%$ with emission wavelength over $664 \mathrm{~nm}$.

The energy gap law ${ }^{1,2}$, simply speaking as the fact that nonradiative decay will significantly increase with the decreased energy gap between zero-vibration levels ( $\Delta$ Ev $v_{0-0}$ ) of the lowest lying singlet $\left(S_{1}\right)$ or triplet $\left(T_{1}\right)$ states and the ground state $\left(S_{0}\right)$, has hindered the development of deep

\footnotetext{
${ }^{1}$ Key Lab of Organic Optoelectronics and Molecular Engineering of Ministry of Education, Department of Chemistry, Tsinghua University, Beijing 100084, China. ${ }^{2}$ Center for Flexible Electronics Technology, Tsinghua University, Beijing 100084, China.

*E-mail: ddzhang@mail.tsinghua.edu.cn; duanl@mail.tsinghua.edu.cn
} 
red (DR)/ near-infrared (NIR) organic emitters for decades, despite their great demanding for applications in night vision, biomedical imaging, optical communications and computing ${ }^{3-8}$. To be specified, in the absence of a zero-order surface crossing, vibronic coupling happens between $v_{0} \mathrm{~S}$ of the excited states $\left(\mathrm{S}_{1}\right.$ or $\left.\mathrm{T}_{1}\right)$ and the higher vibration levels $\left(v_{\mathrm{n}}\right)$ of $\mathrm{S}_{0}$ state (Fig. 1a). And the smaller the $\Delta E v_{0-0}$ of $S_{1}$ and $S_{0}$ is, the enlarged possibility of vibrational wavefunction overlap can be anticipated, exponentially accelerating the radiative-less transitions probability ${ }^{9,10}$. This effect was particularly hold for rigid systems as it was indeed firstly observed for the nonradiative deactivation of polycyclic aromatic hydrocarbons (PAHs) ${ }^{11}$. In those large conjugation PAH structure as exemplified by DBP in Fig. 1b, the highest occupied and lowest unoccupied molecular orbitals (HOMO and LUMO, respectively) of a polycyclic aromatic compound are primarily localized between atoms, forming $\pi$-bonds with bonding/antibonding character. The resulted interactions between the electronic and nuclear vibrational motion will induce $\mathrm{C}=\mathrm{C}$ torsion or $\mathrm{C}$ $\mathrm{H}$ vibration with high frequency. The resulting high energy $v_{\mathrm{n}}$ of $\mathrm{S}_{0}$ state facilitates the vibrational coupling with $v_{0}$ of excited states, and thus enhance the energy gap law effect. Considering that rigid molecular structures were usually adopted for most organic deep red emitters, which exhibit only limited vibrational relaxation at $\mathrm{S}_{1}$ states, the nonradiative transition probability is thereof dominated by the above high vibrational frequency (Supplementary Fig. 1 and Supplementary Tables 1-2). 


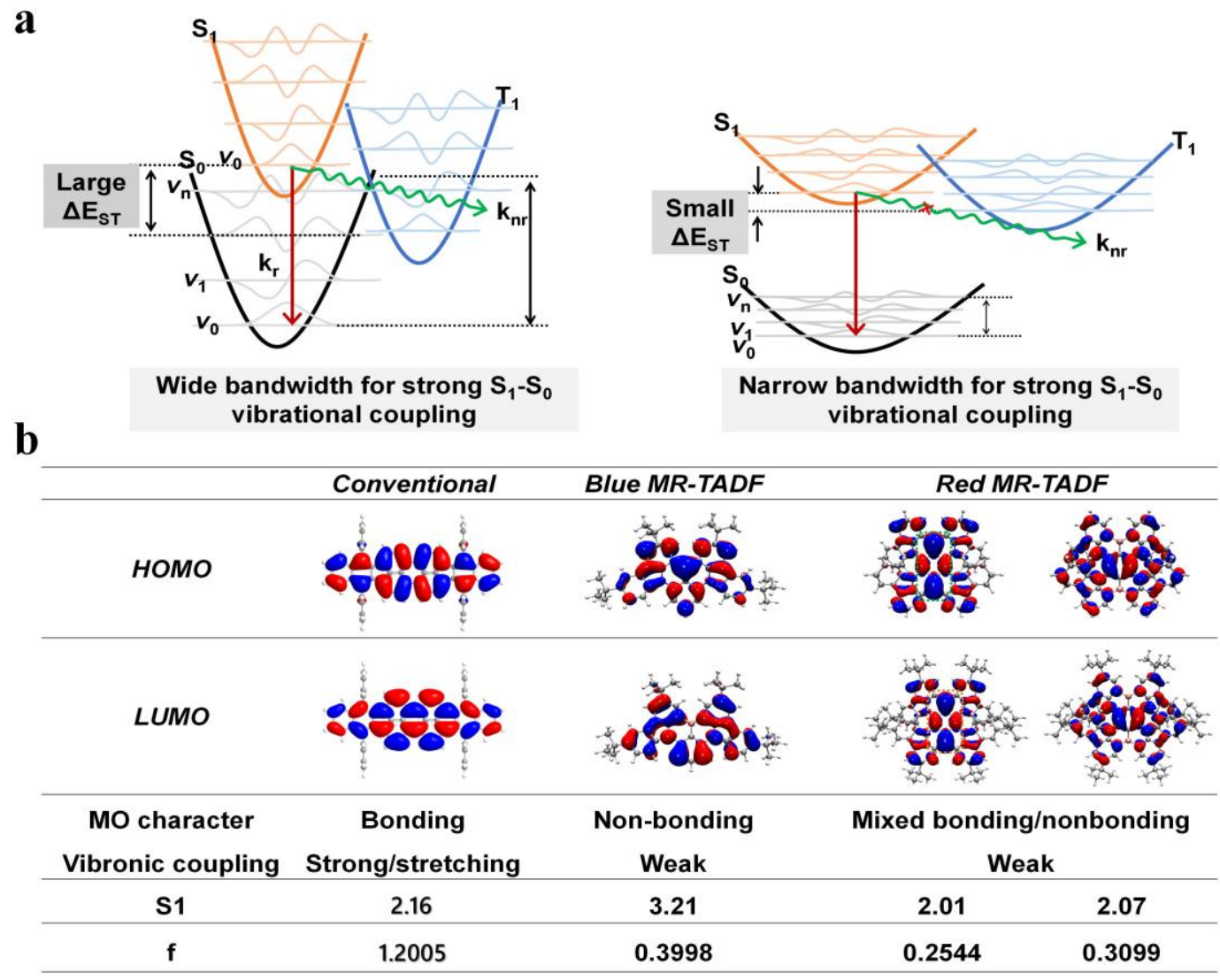

Fig. 1 | New design for beating the limitation of energy gap law. a, Mechanism of the limited vibrational relaxation at $S_{1}$ states by using emitters with a narrow energy-bandwidth as well as small energy gap $\left(\Delta \mathrm{E}_{\mathrm{ST}}\right)$ between $\mathrm{S}_{1}$ and $\mathrm{T}_{1}$ levels. b, HOMO and LUMO of a conventional red emitter (DBP), blue MR-TADF emitter (BCz-BN) and proposed DR/NIR emitters (R-BNs).

Strategies to overcome the limitation of energy gap law has been ongoing pursuits for the development of organic DR/NIR emitters with emission peaks > $650 \mathrm{~nm}$ and still face formidable challenges. Perdeuterated or per-fluorinated approaches have been employed to decrease the number of high-frequency vibrations associated with $\mathrm{C}-\mathrm{H}, \mathrm{O}-\mathrm{H}$ and $\mathrm{N}-\mathrm{H}$ stretching, which however, only showed partial or even negligible improvements ${ }^{12}$. Breakthroughs have recently made by Pt complexes, which show small or negligible interactions between individual molecules 
in the ground state, but dimers or oligomers form on excitation, a shallow or even repulsive potential energy surface can be created in the ground state, theoretically suppressing high frequency vibrational quenching ${ }^{12-15}$. This conceptually advancements have leads to NIR-emitters with impressive PLQY of $80 \%$ under emission peaks of $740 \mathrm{~nm}$ and thus high performance NIROLEDs.

Quite recently, a new class of a fused planar polycyclic aromatic framework with parapositioned electron donating $\mathrm{N}$-atom and electron deficient $\mathrm{B}$-atom have been developed, which induce complementary resonance effects for the offset electron density distributions of HOMO and LUMO orbitals by one atom in an alternating pattern ${ }^{16-24}$. This unique MR effect not only features a short-range reorganization of the electron density between excited states and ground states for a small vibrational relaxation of $S_{1}$, and also minimizes the bonding/antibonding character between adjacent atoms to lower the vibration frequency. Taking advantages of this, a blue emitter with an extremely sharp photoluminescence (PL) band with FWHM of $14 \mathrm{~nm}$ has been obtained ${ }^{16}$. From another perspective, the low vibration frequency also benefits to suppress the vibrational coupling between $v_{\mathrm{n}}$ of $\mathrm{S}_{0}$ and $v_{0}$ of $\mathrm{S}_{1}$ state, showing viable potential to beat the limitation of energy gap law. Also, a bonus can be anticipated that the alternated distribution of HOMO and LUMO render this compound small $\triangle \mathrm{E}_{\mathrm{ST}}$ for thermally activated delayed fluorescence (TADF). This so-called MR-TADF emitters benefit to harness all excitons formed under electrical excitation.

It is thus anticipated that MR-TADF emitters should perform a high photophysical and electrical performances even in the deep red or NIR region. Nevertheless, to the best of knowledge, no DR/NIR MR-TADF emitter has been reported until now. For organic emitters, enlarging conjugation degree is one effective way for redshifted emission. However, the non-bonding 
character in MR-TADF limit the increasement of conjugation facilitate large energy gap for deep blue emission. MR-TADF emitters with enlarged planar structures have been widely reported, yet only realized blue or sky-blue emission ${ }^{16,19}$. Another strategy may be enhancing CT character for TADF emitters but only limited numbers of green MR-TADF emitters have been developed with this strategy, not to mention red or deep red ones ${ }^{22-24}$. Also, the enhanced CT character may break the MR effect and thus broaden emission. Therefore, how to unlock the design strategy for DR/NIR MR-TADF emitters is highly desired.

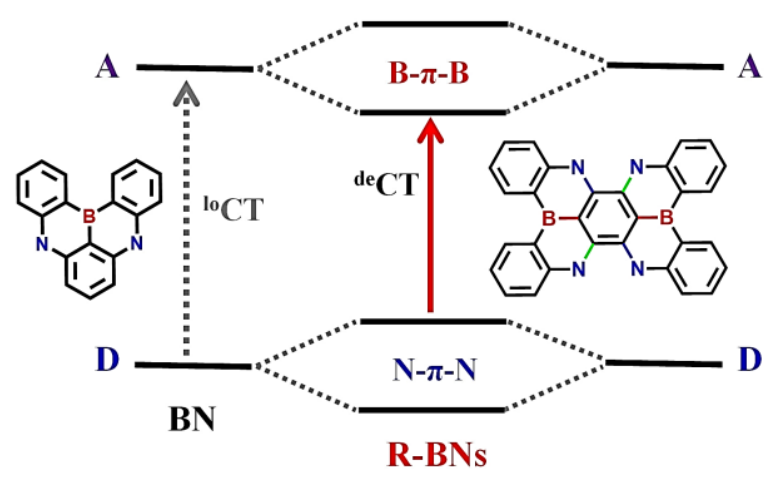

\section{Fig. 2 | The molecular design strategy for DR/NIR MR-TADF emitters.}

Herein, a novel BN-containing skeleton featuring linear $\mathrm{N}-\pi-\mathrm{N}$ and $\mathrm{B}-\pi-\mathrm{B}$ structure is developed (Fig. 2), establishing partial bonding/antibonding character on phenyl core for enhanced electronics coupling of para-positioned $\mathrm{B}$ atoms as well as $\mathrm{N}$ atoms to narrow energy gaps. Also, the remained MR effect on the peripheral skeleton to maintain the MR effect to minimize the bonding/ antibonding character and suppress vibrational coupling between $S_{0}$ and $S_{1}$, thereby fundamentally overcoming the luminescent boundary set by the energy gap law. The target molecules R-BN and R-TBN exhibited extremely high PLQYs of 100\% with emission wavelengths at 664 and $686 \mathrm{~nm}$, respectively. The narrow FWMHs of $38 \mathrm{~nm}$ observed also testify the effectiveness of vibronic suppression. The corresponding OLEDs afford record-high EQEs 
over $28 \%$ with emission wavelength over $666 \mathrm{~nm}$. This molecular design strategy here opens a new avenue towards energy-gap-law free MR-TADF compounds.

a

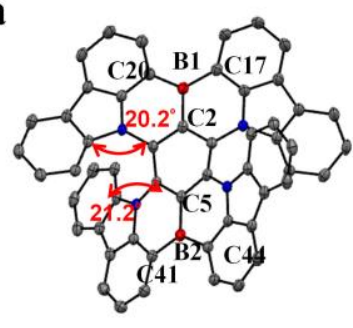

c

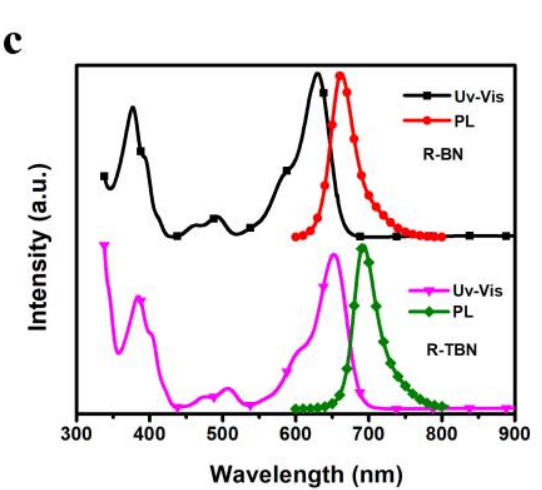

b

B1-C2: 1.546 (2) $\AA$ B1-C17: 1.544 (3) $\AA$ B1-C20: 1.543 (3) $\AA$ B2-C5: 1.553 (2) $\AA$ B2-C41: 1.546 (2) $\AA$ B2-C44: 1.539 (2) $\AA$

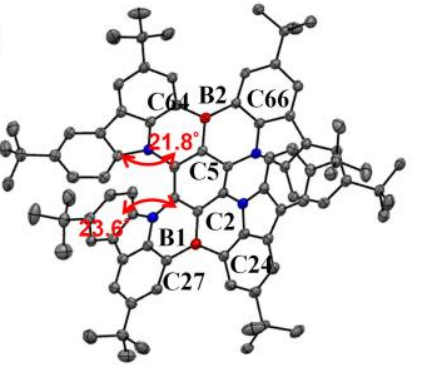

B1-C2: 1.545 (3) $\AA$ B1-C24: 1.545 (3) $\AA$ $B 1-C 27: 1.540$ (3) A B2-C66: 1.535 (3) A B2-C64: 1.540 (4) $\AA$ B2-C5: $1.550(3) \AA$

d

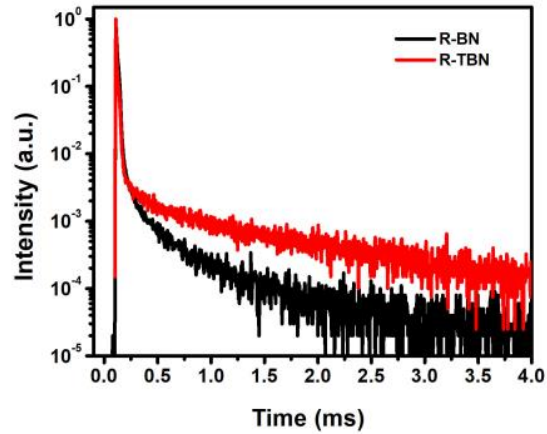

Fig. 3 Structure and photophysical properties of R-BNs. ORTEP drawing of R-BN (a) and R-TBN (b) obtained by X-ray crystallographic analysis. Thermal ellipsoids are shown at 50\% probability. Hydrogen atoms are omitted for clarity. c, UV/Vis absorption, fluorescence (298 K), and phosphorescence $(77 \mathrm{~K})$ spectra of R-BN and R-TBN in toluene. d, Transient PL decay spectra of R-BN/R-TBN doped into CBP films (3 wt\%) at room temperature.

Table 1 | Summary of the photophysical properties of R-BNs.

\begin{tabular}{|c|c|c|c|c|c|c|c|c|c|c|}
\hline & $\begin{array}{l}\mathrm{S}_{1}{ }^{\mathrm{a})} \\
{[\mathrm{eV}]}\end{array}$ & $\begin{array}{l}\mathrm{T}_{1}^{\mathrm{a})} \\
{[\mathrm{eV}]}\end{array}$ & $\begin{array}{l}\Delta \mathrm{EsT}^{\mathrm{a})} \\
{[\mathrm{eV}]}\end{array}$ & $\begin{array}{c}\text { FWHM }^{\text {a) }} \\
{[\mathrm{nm}]}\end{array}$ & $\begin{array}{c}\mathrm{HOMO}^{\mathrm{b})} \\
{[\mathrm{eV}]}\end{array}$ & $\begin{array}{c}\text { LUMO }^{c)} \\
{[\mathrm{eV}]}\end{array}$ & PLQY $^{d)}$ & $\begin{array}{l}\tau_{\mathrm{PF}}{ }^{\mathrm{d})} \\
{[\mathrm{ns}]}\end{array}$ & $\begin{array}{l}\tau_{\mathrm{DF}}{ }^{\mathrm{d})} \\
{[\mathrm{ms}]}\end{array}$ & $\begin{array}{c}k_{\mathrm{r}}^{\mathrm{d})} \\
\left(10^{7} \mathrm{~s}^{-1}\right)\end{array}$ \\
\hline R-BN & 1.87 & 1.69 & 0.18 & 38 & -4.76 & -3.06 & $100 \%$ & 4.7 & 0.31 & 17 \\
\hline R-TBN & 1.79 & 1.63 & 0.16 & 38 & -4.69 & -3.00 & $100 \%$ & 10.3 & 0.71 & 7.1 \\
\hline
\end{tabular}


${ }^{a}$ Measured in the toluene solution with a concentration of $10^{-5} \mathrm{~mol} / \mathrm{L}$. ${ }^{b}$ Measured in dry dichloromethane with a concentration of $10^{-3} \mathrm{~mol} / \mathrm{L}$. ${ }^{\mathrm{c}}$ Measured in dry $\mathrm{N}, \mathrm{N}$-dimethylformamide with a concentration of $10^{-3} \mathrm{~mol} / \mathrm{L}$. ${ }^{\mathrm{d}}$ Measured in $3 \mathrm{wt} \%$-doped films of $\mathrm{R}-\mathrm{BNs}$ in CBP.

R-BNs were synthesized in two steps from commercially available starting materials. Palladium or other rare-earth-metal catalysts were not required, making R-BNs cost effective. The single crystal X-ray diffraction analyses demonstrated that R-BN combines the characteristics of a fusion structure and a sterically hindered structure, where small torsional angles $\left(\sim 20^{\circ}\right)$ of the carbazolyl groups could be found (Fig. 3a,b and Supplementary Fig. 2). This limited torsional flexibility can be another major factor in reducing the non-radioactive decay of R-BNs. The photophysical properties of R-BNs are listed in Table 1. The UV-vis absorption spectra measured in toluene exhibited a strong absorption band corresponding to the HOMO-LUMO transition with a maximum at $629 \mathrm{~nm}(\log \varepsilon=5.13$, where $\varepsilon$ is the molar excitation coefficient) for R-BN and 651 $\mathrm{nm}(\log \varepsilon=4.87)$ for R-TBN (Fig. 3c and Supplementary Fig. 3). Compared with BCz-BNs, the significantly reduced energy gap (Eg) and high $\varepsilon$ in R-BNs could be assigned to the efficient energy splitting in molecular orbitals (MOs), cleverly avoiding the undesired quenching process produced by the introduction of strong CT. Time-dependent density functional theory (TD-DFT) calculations and cyclic voltammetry (CV) measurements revealed this new skeleton with linear $\mathrm{N}-\pi-\mathrm{N}$ and $\mathrm{B}-\pi-\mathrm{B}$ structure exhibited a much shallower HOMO as well a deeper LUMO than that of $\mathrm{BCz}-\mathrm{BN}$, which is essentially different to the blue emissive $v$-DABNA with meta $\mathrm{N}-\pi-\mathrm{N}$ and $\mathrm{B}-\pi-\mathrm{B}$ characteristics (Supplementary Figs. 4-5). Moreover, the orbital distributions of R-BN and R-TBN illustrated a unique mixed bonding and nonbonding characters. On the core phenyl plane, $\pi$-bonds with bonding/antibonding character were observed, assigned to the electronic coupling between $\mathrm{B}$ atoms or $\mathrm{N}$ atoms in para-positions. In that scenario, delocalized excited states can be anticipated ${ }^{25,26}$. The fluorescence spectra at $298 \mathrm{~K}$ exhibited strong and sharp emission 
bands at 662 and $692 \mathrm{~nm}$. The PLQYs of R-BNs were extremely high (100\% for R-BN and RTBN), the FWHMs and Stokes shifts were very small (38 and $33 \mathrm{~nm}$ for R-BN, 38 and $41 \mathrm{~nm}$ for R-TBN)-indicating that the non-bonding HOMO and LUMO minimize the vibronic coupling between the $S_{0}$ and $S_{1}$ states and the vibrational relaxation at the $S_{1}$ state to suppress the nonradiative decay. This is well supported by the TD-DFT calculations (Supplementary Figs. 6-9 and Supplementary Table 3), where a high degree of electron-hole overlap could be found, indicating that the Franck-Condon excitation between $S_{0}$ and $S_{1}$ has a high oscillator strength ( $\mathrm{f}=0.25$ for $\mathrm{R}-\mathrm{BN}$ and 0.33 for $\mathrm{R}-\mathrm{TBN}$ ). Additionally, the geometric changes of molecular conformation between $S_{0}$ and $S_{1}$ states and structural relaxation at the $S_{1}$ state are extremely small with reorganization $\left(\lambda_{\mathrm{S}}\right) /$ structural relaxation $\left(\lambda_{\mathrm{s}}{ }^{*}\right)$ energies were calculated to be merely 0.11 and $0.12 \mathrm{eV}, 0.10$ and $0.11 \mathrm{eV}$ for R-BN and R-TBN, respectively, significantly smaller than typical DR/NIR organic molecules $(0.26-0.52 \mathrm{eV})$. Based on the fluorescence and phosphorescence spectra recorded in a frozen toluene $(77 \mathrm{~K})$ matrix (Supplementary Fig. 10), the $\Delta \mathrm{E}_{\mathrm{ST}}$ were calculated to be $0.18 \mathrm{eV}$ for R-BN and $0.16 \mathrm{eV}$ for R-TBN. Such small $\Delta \mathrm{E}_{S T}$ values are favorable for exciton up-conversion from $T_{1}$ to $S_{1}$ at ambient condition. These are promising characteristics for constructing DR/FR dyes with high-efficiency.

The photophysical properties of R-BNs in doped films were measured with 4,4'-di(9H-carbazol9-yl)-1,1'-biphenyl (CBP) as the wide-energy gap host. As depicted in Figure 3, both the doped films (dopant concentration of $3 \mathrm{wt} \%$ ) gave DR/NIR emissions with emission maxima and FWHMs of 672 and $48 \mathrm{~nm}$ for R-BN, 698 and $49 \mathrm{~nm}$ for R-TBN, respectively (Supplementary Fig. 11). Owing to the special rigid MR structures, the corresponding PLQYs were also measured to be $100 \%$, which represent the highest results among reported DR/NIR emitters, suggesting the effective suppression of the quenching process. The TADF characteristics of the R-BNs were 
recorded and are displayed in Supplementary Fig. 12. Both prompt and delayed fluorescence components were clearly identified, which can be unambiguously assigned to the TADF emission. The quantum yields $\left(\Phi_{\mathrm{F}}=0.82\right.$ and $\Phi_{\mathrm{DF}}=0.18$ for $\mathrm{R}-\mathrm{BN}, \Phi_{\mathrm{F}}=0.73$ and $\Phi_{\mathrm{DF}}=0.27$ for $\left.\mathrm{R}-\mathrm{TBN}\right)$ and lifetimes $\left(\tau_{\mathrm{F}}=4.7 \mathrm{~ns}\right.$ and $\tau_{\mathrm{DF}}=0.31 \mathrm{~ms}$ for $\mathrm{R}-\mathrm{BN}, \tau_{\mathrm{F}}=10.3 \mathrm{~ns}$ and $\tau_{\mathrm{DF}}=0.71 \mathrm{~ms}$ for $\left.\mathrm{R}-\mathrm{TBN}\right)$ of the fluorescence and TADF components were determined based on the total $\Phi$ and ratio of the integrated area of each component in the transient spectra to the total integrated $\operatorname{area}^{27}$. Based on these quantum yields and lifetimes, the rate constants of fluorescence $\left(k_{\mathrm{F}}\right)$, intersystem crossing $\left(k_{\mathrm{ISC}}\right)$ and reverse intersystem crossing $\left(k_{\mathrm{RISC}}\right)$ were calculated to be $1.7 \times 10^{8}, 3.9 \times 10^{7}$ and $3.9 \times$ $10^{3} \mathrm{~s}^{-1}$ for R-BN, $7.1 \times 10^{7}, 2.6 \times 10^{7}$ and $1.9 \times 10^{3} \mathrm{~s}^{-1}$ for R-TBN, respectively, using the methodology provided in the literature ${ }^{28}$. The large $k_{\mathrm{F}}$ values are consistent with the large molar excitation coefficient $\left(\log \varepsilon=5.13\right.$ and 4.87) and oscillator strengths for the $\mathrm{S}_{1}-\mathrm{S}_{0}$ transition calculated by TD-DFT ( $\mathrm{f}=0.22$ and 0.27 , Supplementary Table 3 ). These are promising characteristics of TADF dye for fabricating FR/NIR OLED with high efficiency and color-purity.

To demonstrate the potential of the present DR/NIR emitters, devices with the following structure were fabricated: indium tin oxide (ITO); 1,4,5,8,9,11-hexaazatriphenylene hexacarbonitrile (HATCN, $10 \mathrm{~nm}$ ); 1,1-bis[(di-4-tolylamino)phenyl]cyclohexane (TAPC, $60 \mathrm{~nm}$ ); tris(4-carbazolyl-9-ylphenyl)amine (TCTA, $10 \mathrm{~nm}$ ); CBP:30wt\% Ir(mphmq)2tmd:3wt\%R-BNs (30 nm); 4,6-bis(3-(9H-carbazol-9-yl)phenyl)pyrimidine (CzPhPy, $10 \mathrm{~nm}) ; \quad 4,6-\mathrm{Bis}(3,5-$ di(pyridin-4-yl)phenyl)-2-methylpyrimidine (B4PyMPM, $50 \mathrm{~nm})$; LiF (0.5 nm); Al (150 nm). The EL characteristics and the ionization potentials and electron affinities of these devices are shown in Fig. 4 and Supplementary Figs. 13-14; the OLEDs based on R-BN and R-TBN exhibit stable DR/NIR emissions with peaks at 664 and $686 \mathrm{~nm}$ and small FWHMs of 48 and $49 \mathrm{~nm}$, respectively. The corresponding CIE coordinates are $(0.719,0.280)$ and $(0.721,0.278)$, representing the reddest 
emissions ever reported in OLEDs ${ }^{4,29-39}$. The maximum EQE values were $28.4 \%$ for R-BN and $28.1 \%$ for R-TBN, respectively. To our knowledge, the EQEs here are the record-high values among all DR/NIR OLEDs ${ }^{4,29-39}$. More importantly, the maximum radiances of $6.5 \times 10^{5}$ and 7.3 $\times 10^{5} \mathrm{~mW} \mathrm{sr}^{-1} \mathrm{~m}^{-2}$ for R-BN and R-TBN devices also set new records for efficient DR/NIR OLEDs $(\mathrm{EQE}>5 \%)^{33}$.
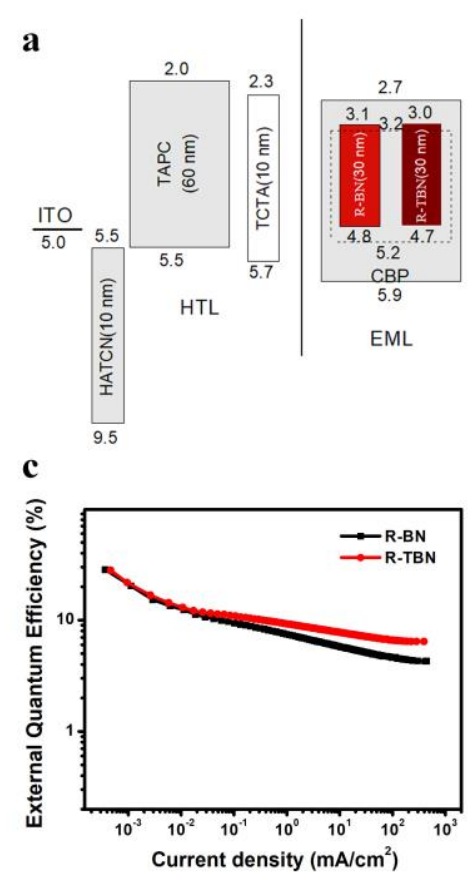
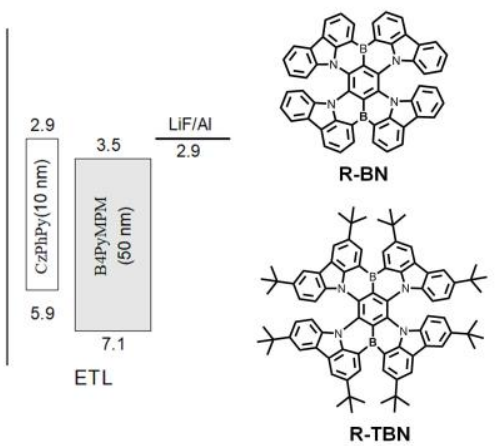

d

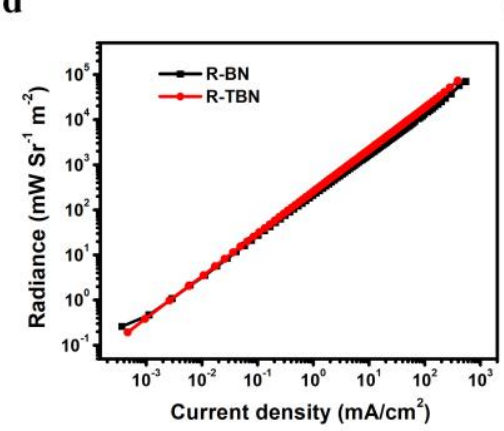

b

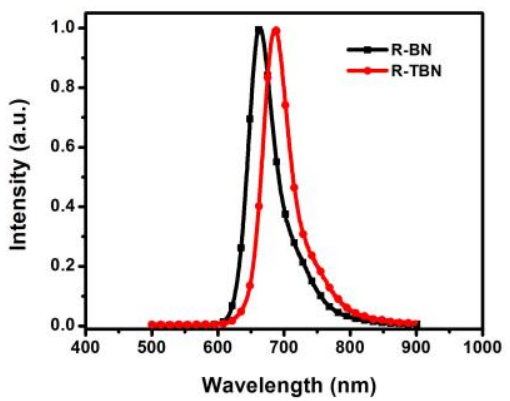

e

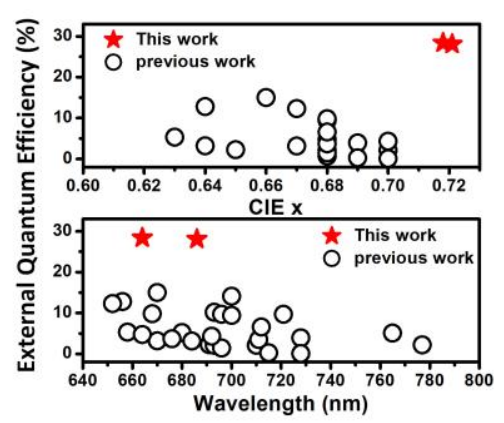

Fig. 4 | OLED performance. a, The energy-level diagrams and the emitter structures of the devices. b, The EL spectra of the optimized DR/NIR devices. c, EQE versus current density characteristics. d, Radiance versus current density characteristics. e, CIE x (up)/EQE (down) summary of DR/NIR TADF OLEDs with emission peak > $650 \mathrm{~nm}$ (for references, see Supplementary Table 5).

In summary, a rational molecular design strategy for efficient DR/NIR MR emitters was demonstrated through energy splitting, and it is shown that the new BN-containing skeleton with 
linear $\mathrm{N}-\pi-\mathrm{N}$ and $\mathrm{B}-\pi-\mathrm{B}$ structure could significantly redshift the emission. A proof of concept is provided by a series of new DR/NIR-emitting MR emitters, where the special localization of the HOMO and LUMO in different atoms could minimize bonding/ antibonding character and suppress vibrational coupling and relaxation, providing highly efficient DR/NIR emission (PLQY 100\%). On this basis, we successfully demonstrate outstanding DR/NIR OLEDs achieving record EQEs of more than $28 \%$ with EL peaks at 664 and $686 \mathrm{~nm}$. In addition, the corresponding CIE coordinates of $(0.719,0.280)$ and $(0.721,0.278)$ also represent the reddest emissions ever reported in OLEDs. We believe our strategy would continue to guide the exploration of highly efficient DR/NIR emitters and corresponding highly efficient OLEDs with high color purity.

\section{References}

1. Caspar, J. V. \& Meyer, T. J. Application of the energy gap law to nonradiative, excited-state decay. J. Phys. Chem. 87, 952-957 (1983).

2. Caspar, J. V. et al. Application of the energy gap law to the decay of charge-transfer excited states. J. Am. Chem. Soc. 104, 630-632 (1982).

3. Takahiko, Y. et al. A. Near-infrared organic light-emitting diodes for biosensing with high operating stability. Appl. Phys. Express.10, 074101 (2017).

4. Yuan, Y. et al. Over 10\% EQE near-infrared electroluminescence based on a thermally activated delayed fluorescence emitter. Adv. Funct. Mater. 1700986 (2017).

5. Tessler, N. et al. Efficient near-infrared polymer nanocrystal light-emitting diodes. Science 295, 1506-1508 (2002). 
6. Zhu, S. et al. Repurposing cyanine NIR-I dyes accelerates clinical translation of nearinfrared- II (NIR- II) bioimaging. Adv. Mater. 30, 1802546 (2018).

7. Antaris, A. L. et al. A small-molecule dye for NIR-II imaging. Nat. Mater. 15, 235-242 (2016).

8. Wang, D. et al. Highly efficient photosensitizers with far- red/near-infrared aggregationinduced emission for in vitro and in vivo cancer theranostics. Adv. Mater. 30, 1802105 (2018).

9. Siebrand, W. Radiationless transitions in polyatomic molecules. II: triplet-ground-state transitions in aromatic hydrocarbons. J. Chem. Phys. 47, 2411-2422 (1967).

10. Englman, R. \& Jortner, J. The energy gap law for radiationless transitions in large molecules. Mol. Phys. 18, 145-164 (1970).

11. Zampetti, A. et al. Near-Infrared (NIR) Organic Light-Emitting Diodes (OLEDs): Challenges and Opportunities. Adv. Funct. Mater. 29, 1807623 (2019).

12. Ly, K. T. et al. Near-infrared organic light-emitting diodes with very high external quantum efficiency and radiance. Nat. Photon. 11, 63-68 (2017).

13. Klaus, D. R. et al. 1D polymeric platinum cyanoximate: a strategy toward luminescence in the near-infrared region beyond 1,000 nm. Inorg. Chem. 54, 1890-1900 (2015).

14. Zhang, Y. et al. Achieving NIR emission for donor-acceptor type platinum(ii) complexes by adjusting coordination position with isomeric ligands. Inorg. Chem. 57, 14208-14217 (2018). 
15. Wei, Y. C. et al. Overcoming the energy gap law in near-infrared OLEDs by excitonvibration decoupling. Nat. Photon. 14, 570-577 (2020).

16. Yasuhiro, K. et al. Narrowband deep-blue organic light-emitting diode featuring an organoboron-based emitter. Nat. Photon. 13, 678-682 (2019).

17. Hatakeyama, T. et al. Ultrapure blue thermally activated delayed fluorescence molecules: efficient HOMO-LUMO separation by the multiple resonance effect. Adv. Mater. 28, 27772781 (2016);

18. Nakatsuka, S., Gotoh, H., Kinoshita, K., Yasuda, N. \& Hatakeyama, T. Divergent synthesis of heteroatom-centered 4,8,12-triazatriangulenes. Angew. Chem. Int. Ed. 56, 5087-5090 (2017).

19. Matsui, K. et al. One-shot multiple borylation toward BN-doped nanographenes. J. Am. Chem. Soc. 140, 1195-1198 (2018).

20. Liang, X. et al. Peripheral amplification of multi-resonance induced thermally activated delayed fluorescence for highly efficient OLEDs. Angew. Chem. Int. Ed. 57, 11316-11320 (2018).

21. Knöller, J. A. et al. Berichtigung: Divergente Synthese von B, N, B-Benzo [4] helicenen durch intramolekulare Borylierung unter sequenzieller B-Mes- Bindungsspaltung. Angew. Chem. Int. Ed. 132, 3181-3185 (2020).

22. Xu, Y. et al. Constructing Charge-Transfer Excited States Based on Frontier Molecular Orbital Engineering: Narrowband Green Electroluminescence with High Color Purity and Efficiency. Angew. Chem. Int. Ed. 59, 17442-17446 (2020). 
23. Zhang, Y. et al. Multi - Resonance Induced Thermally Activated Delayed Fluorophores for Narrowband Green OLEDs. Angew. Chem. Int. Ed. 58, 16912 (2019).

24. Zhang, Y. et al. Achieving Pure Green Electroluminescence with CIEy 0.69 and EQE $28.2 \%$ from a Novel Aza-Fused Multi-Resonance Emitter. Angew. Chem. Int. Ed. 59, 17499 (2020).

25. Noda, H. et al. Critical role of intermediate electronic states for spin-flip processes in chargetransfer-type organic molecules with multiple donors and acceptors. Nat. Mater. 18, 10841090 (2019).

26. Zhang, D. et al. Efficient and Stable Deep-Blue Fluorescent Organic Light- Emitting Diodes Employing a Sensitizer with Fast Triplet Upconversion. Adv. Mater. 32, 1908355 (2020).

27. Park, I. S. et al. High-performance dibenzoheteraborin-based thermally activated delayed fluorescence emitters: molecular architectonics for concurrently achieving narrowband emission and efficient triplet-singlet spin conversion. Adv. Funct. Mater. 28, 1802031 (2018).

28. Zhang, D. et al. Versatile Indolocarbazole- Isomer Derivatives as Highly Emissive Emitters and Ideal Hosts for Thermally Activated Delayed Fluorescent OLEDs with Alleviated Efficiency Roll- Off. Adv. Mater. 30, 1705406 (2018).

29. Furue, R. et al. Highly Efficient Red-Orange Delayed Fluorescence Emitters Based on Strong $\pi$-Accepting Dibenzophenazine and Dibenzoquinoxaline Cores: toward a Rational Pure-Red OLED Design. Adv. Opt. Mater. 6, 1701147 (2018).

30. Li, C. et al. Deep-red to near-infrared thermally activated delayed fluorescence in organic solid films and electroluminescent devices. Angew. Chem. Int. Ed. 56, 11525-11529 (2017). 
31. Wang, S. et al. Highly efficient near-infrared delayed fluorescence organic light emitting diodes using a phenanthrene-based charge-transfer compound. Angew. Chem. Int. Ed. 54, 13068-13072 (2015).

32. Sun, K. et al. Near-infrared thermally activated delayed fluorescent dendrimers for the efficient non-doped solution-processed organic light-emitting diodes. Org. Electron. 48, 389-396 (2017).

33. Kim, D. et al. High-efficiency electroluminescence and amplified spontaneous emission from a thermally activated delayed fluorescent near-infrared emitter. Nat. Photon. 12, 98104 (2018).

34. Xue, J. et al. Highly Efficient Thermally Activated Delayed Fluorescence via J-Aggregates with Strong Intermolecular Charge Transfer. Adv. Mater. 31, 1808242 (2019).

35. Chen, J. X. et al. Red/Near-Infrared Thermally Activated Delayed Fluorescence OLEDs with Near 100\% Internal Quantum Efficiency. Angew. Chem. Int. Ed. 58,14660-14665 (2019).

36. Zhao. B. et al. Highly Efficient Deep-Red Non-Doped Diodes Based on a T-Shape Thermally Activated Delayed Fluorescence Emitter. Angew. Chem. Int. Ed. 59, $19042-$ 19047 (2020).

37. Zhang, Y. et al. Achieving High-Performance Solution-Processed Deep-Red/Near-Infrared Organic Light-Emitting Diodes with a Phenanthroline-Based and Wedge-Shaped Fluorophore. Adv. Electron. Mater. 5, 1800677 (2019).

38. Yang, T. et al. Construction of Efficient Deep-Red/Near-Infrared Emitter Based on a Large $\pi$-Conjugated Acceptor and Delayed Fluorescence OLEDs with External Quantum Efficiency of over 20\%. J. Phys. Chem. C 123, 18585-18592 (2019). 
39. Kumsampao, J. et al. A Simple and Strong Electron-Deficient 5, 6-Dicyano [2, 1, 3] benzothiadiazole-Cored Donor-Acceptor-Donor Compound for Efficient Near Infrared Thermally Activated Delayed Fluorescence. Chem. - Asian J. 15, 3029-3036 (2020).

\section{Acknowledgements ((optional))}

This work was supported by the National Key Basic Research and Development Program of China (Grant No. 2016YFB0401003), the National Science Fund of China (Grant Nos. 51525304, and 61890942), the China Postdoctoral Science Foundation (Grant No. 2019M650628) and Foshan Xianhu Laboratory of the Advanced Energy Science and Technology Guangdong Laboratory XHT2020-005.

\section{Competing interests}

The authors declare no competing interests. 